\title{
Clamping down on calpains to treat injury-induced spasticity
}

Following spinal cord injury (SCI), spasticity resulting from hyperexcitability of motor neurons is common. A recent study has shown that calpain-mediated cleavage of sodium channels in motor neurons contributes to this complication of SCI and provides evidence that pharmacological inhibition of a calpain could be a useful therapeutic strategy for such spasticity.

Upregulation of the persistent sodium current $\left(\mathrm{I}_{\mathrm{NaP}}\right)$ underlies much of the increased excitability of motor neurons after SCI. To investigate the mechanisms that link SCI with $\mathrm{I}_{\mathrm{NaP}}$, the authors of the current study performed spinal cord lesion in rodents followed by immunohistochemistry of spinal cord samples. Compared with sham-operated rodents, SCI was associated with upregulation of the voltagegated sodium channel $\mathrm{Na}_{\mathrm{v}} 1.6$ in motor neurons.

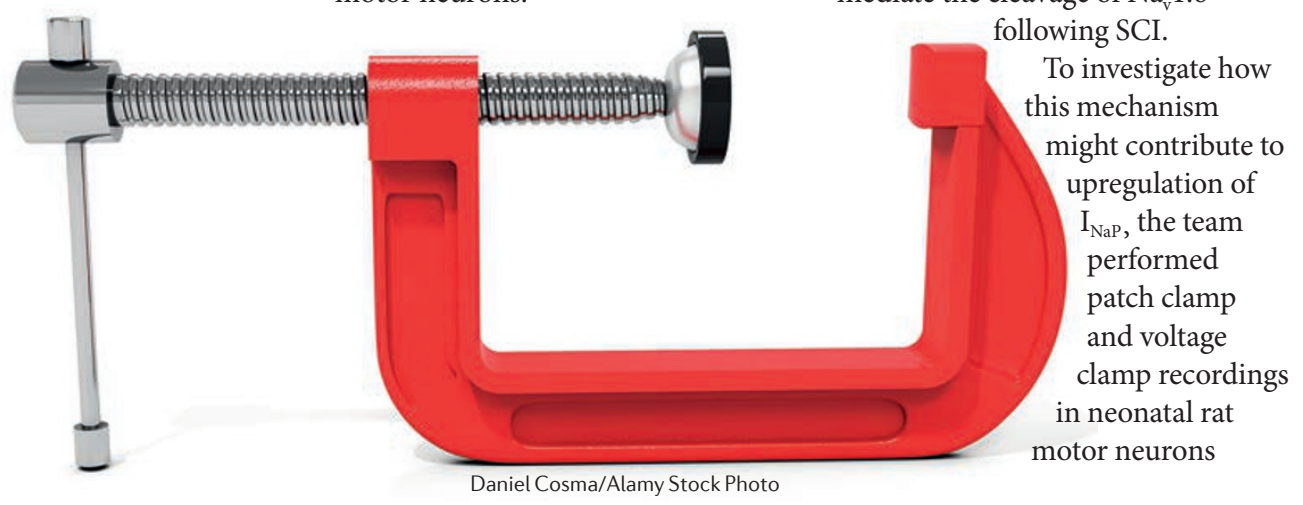

Next, the authors carried out further analysis of $\mathrm{Na}_{\mathrm{v}} 1.6$ expression using western blotting on the membrane fractions of rodent motor neurons. A $\mathrm{Na}_{\mathrm{v}}$-specific antibody revealed an $\sim 250 \mathrm{kDa}$ band consistent with full-length $\mathrm{Na}_{\mathrm{v}}$. Notably, although the intensity of the $\sim 250 \mathrm{kDa}$ band was unchanged by SCI, an $\sim 120 \mathrm{kDa}$ cleavage product was strongly upregulated.

In adult rat spinal cord homogenates, addition of calcium generated a similar $\sim 120 \mathrm{kDa}$ band, suggesting the involvement of a calcium-dependent protease, which the investigators posited could be a calpain. To test this hypothesis, they pretreated the spinal cord homogenates with MDL28170, a calpain inhibitor, and indeed saw no formation of an $\sim 120 \mathrm{kDa}$ band in response to calcium. Together, these findings suggest that calpains mediate the cleavage of $\mathrm{Na}_{\mathrm{v}} 1.6$ following SCI. this mechanism might contribute to upregulation of $\mathrm{I}_{\mathrm{NaP}}$, the team performed patch clamp and voltage clamp recordings in neonatal rat Daniel Cosma/Alamy Stock Photo after SCI. Compared with vehicle treatment, intraperitoneal injections of MDL28170 for 8 days following SCI significantly reduced the formation of the $\sim 120 \mathrm{kDa}$ band on western blots and reduced the amplitude and the density of $\mathrm{I}_{\mathrm{NaP}}$. Moreover, calpains similarly increased $\mathrm{I}_{\mathrm{NaP}}$ in HEK293 cells expressing $\mathrm{Na}_{\mathrm{v}} 1.6$.

Finally, in adult rats with SCI, injection of MDL28170 daily for 10 days, beginning 30-60 days after injury, suppressed the cleavage of $\mathrm{Na}_{\mathrm{v}}$ and significantly reduced muscle spasms relative to vehicle-treated SCI rats. Importantly, the beneficial effects of MDL28170 were maintained at 3 weeks after discontinuation of treatment.

The authors noted some residual upregulation of $\mathrm{I}_{\mathrm{NaP}}$ after calpain inhibition with MDL28170, and they suggest that this could be due to mechanisms involving 5-HT receptors, which are known to be upregulated after SCI and to stimulate $\mathrm{I}_{\mathrm{NaP}}$.

This study suggests inhibition of calpains, even long after SCI, could have beneficial effects on subsequent spasticity.

Katie Kingwell

ORIGINAL ARTICLE Brocard, C. et al. Cleavage of $\mathrm{Na}^{+}$channels by calpain increases persistent $\mathrm{Na}^{+}$ current and promotes spasticity after spinal cord injury. Nat. Med. 22, 404-411 (2016) 